\title{
Laboratório de Linguagens Formais -
}

\section{LabLF}

Robson da Silva Aguiar

Laboratório de Informática na Educação da Universidade Federal do Pará (Labie - UFPA).

robson_cbcc@yahoo.com.br
Janne Yukiko Yoshikawa Oeiras

Laboratório de Informática na Educação da Universidade Federal do Pará (Labie - UFPA).

joeiras@ufpa.br

Resumo Este artigo apresenta um ambiente computacional de suporte à aprendizagem de disciplinas de Teoria da Computação, denominado Laboratório de Linguagens Formais (LabLF). Na literatura podem ser encontrados diversos outros ambientes que possibilitam ao aluno criar e testar modelos formais que solucionam problemas computacionais propostos pelo professor. $O$ diferencial do LabLF está no fato de permitir a elaboração de listas de exercícios ou avaliações que podem ser corrigidas de modo automático ou semi-automático.

Palavras-Chave: LabLF, Informática na Educação, JFLAP, Feedback Automático.

\begin{abstract}
This paper presents a computational environment that supports learning of the disciplines of Theory of Computation, called the Laboratory of Formal Languages (LabLF). In the literature can be found several other environments that allow students to create and test formal models that solve computational problems proposed by the teacher. The differential of LabLF is in fact permit the preparation of lists of exercises and assessments that can be corrected automatically or semi-automatic
\end{abstract}

Keywords: LabLF, Education Informatic, JFLAP, Automatic Feedback 


\section{Introdução}

O estudo de Teoria da Computação (TC) envolve disciplinas que se apóiam em um alicerce baseado em teorias e conceitos matemáticos que, para muitos alunos, são difíceis de compreender [8]. Por essa razão, na literatura foram propostas diversas ferramentas computacionais, como as relatadas em $[1,2,3,4,5]$, para auxiliar a aprendizagem de TC.

Em disciplinas de TC, um professor geralmente indica listas de exercícios para os alunos resolverem com o suporte de ferramentas computacionais existentes. Assim, por meio destas ferramentas, os alunos podem construir seus modelos computacionais correspondentes e averiguar a corretude de suas soluções. Portanto, é esperado que o aluno, por meio do manuseio dessas ferramentas computacionais, possa ampliar e consolidar seus conhecimentos e habilidades na resolução de problemas.

Embora tais ferramentas tenham o potencial para ajudar o aluno a compreender o funcionamento dos seus modelos construídos, para cada exercício proposto pelo professor o aluno é quem tem que aprender a testar a corretude de sua solução. Por exemplo, no caso do estudo de Autômatos Finitos o aluno precisa definir um conjunto de palavras que acusem se sua resposta está correta ou não (chamadas a partir de então de palavras de teste), o que nem sempre é uma tarefa fácil para quem está iniciando esse estudo. Assim, essa dificuldade em saber se sua resposta está correta levam o aluno a buscar feedback junto aos monitores ou do professor da disciplina.

Além desses exercícios, a tarefa de correção de avaliações feita pelo professor também é árdua a depender da quantidade de alunos em sua turma. As soluções dos alunos para os problemas propostos nessas avaliações advêm de um processo criativo e muitas vezes são diferentes entre si.

A fim de ajudar os alunos com feedback rápido sobre sua solução a exercícios propostos pelo professor em listas e auxiliar este na tarefa de correção de avaliações é que o Laboratório de Linguagens Formais (LabLF) foi desenvolvido. Este ambiente permite ao professor criar, além de exercícios convencionais: Discursivos, Verdadeiro ou Falso e Múltipla Escolha, exercícios e perguntas em avaliações em que suas soluções são modelos computacionais que o ambiente armazena como gabaritos. Assim, os alunos ao tentarem responder tais exercícios, submetendo uma solução, obtêm feedback rápido que os informa se sua solução é a solução esperada pelo professor ou não.
A seção 2 deste artigo apresenta as ferramentas computacionais de auxílio ao ensino e a aprendizagem de TC que tiveram grande importância na motivação e no desenvolvimento do LabLF.

A seção 3 apresenta o LabLF com ênfase no processo de correção automática de exercícios propostos pelo professor. A seção 4 discorre sobre o uso do LabLF em uma turma real na Universidade Federal do Pará (UFPA). E a seção 5 apresenta as Considerações finais.

\section{Ferramentas computacionais de au- xílio ao ensino e a aprendizagem de TC}

A principal motivação para a criação de ferramentas computacionais de auxílio ao ensino e a aprendizagem de TC é facilitar o processo de aprendizagem dos alunos e poder reduzir as suas dificuldades ao tentarem resolver exercícios com diferentes graus de complexidade.

Na literatura coexistem diversos tipos de ferramentas computacionais voltadas ao estudo de TC, cada uma apropriada às diferentes dificuldades enfrentadas pelos alunos de TC. Algumas ferramentas computacionais são mais indicadas do que outras, pois em princípio todas foram desenvolvidas para auxiliar especificamente em uma dificuldade dos alunos.

Por exemplo, se os alunos apresentarem dificuldades em definir formalmente um modelo computacional (Autômato Finito Determinístico (AFD), Autômato Finito Não-Determinístico (AFND), Gramática Regular (GR), Expressão Regular (ER), Autômato com Pilha (AP), Máquina de Turing (MT) etc.) o professor pode indicar algumas ferramentas computacionais como o Sistema de Criação e Teste de Modelos Formais (SCTMF) [3] e o Language Emulator [4] para ajudá-los a superarem esta dificuldade.

A Tabela 1 organiza algumas ferramentas computacionais encontradas na literatura para o estudo de TC. Para cada uma delas estão marcados (com um "X") os modelos computacionais implementados, a possibilidade de visualizar ou não o diagrama do modelo computacional que se está construindo (Saída gráfica) e a ajuda dada por cada uma destas ferramentas computacionais na dificuldade do aluno em saber se sua solução a um exercício está correta ou não (Correção Automática). 


\begin{tabular}{|c|c|c|c|c|c|c|c|}
\hline & AFD/AFND & GR & ER & AP & MT & Saída Gráfica & Correção Automática \\
\hline GAM & $x$ & & & & & $x$ & \\
\hline VAS & $x$ & & & & $x$ & $x$ & \\
\hline Language Emulator & $x$ & $x$ & $x$ & & & & \\
\hline SCTMF & $x$ & $x$ & $x$ & $x$ & $x$ & & \\
\hline JFLAP & $x$ & $x$ & $x$ & $x$ & $x$ & $x$ & \\
\hline LabLF & $x$ & $x$ & $x$ & $x$ & $x$ & $x$ & $x$ \\
\hline
\end{tabular}

Tabela 1: Ferramentas e seus modelos de computação implementados.

A primeira linha da Tabela 1 apresenta a ferramenta computacional Ginux Abstract Machine (GAM) [1]. Seu uso é restrito ao estudo dos modelos computacionais AFD e AFND e sua principal vantagem é a utilização de interface gráfica que permite ao usuário visualizar o diagrama do modelo computacional e manipulá-lo. A tela principal desta ferramenta computacional pode ser vista na Figura 1.
Além disso, GAM [1] permite o estudo de conversão de AFND para AFD e ainda conta com o processo de minimização deste último. No entanto, não permite ao aluno saber rapidamente se o AFD ou AFND construído como solução a um exercício é realmente a solução correta. O aluno precisa concluir que sua resposta está correta ou não por meio de palavras de teste inseridas por ele na ferramenta e testadas sobre o AFD ou AFND construído.

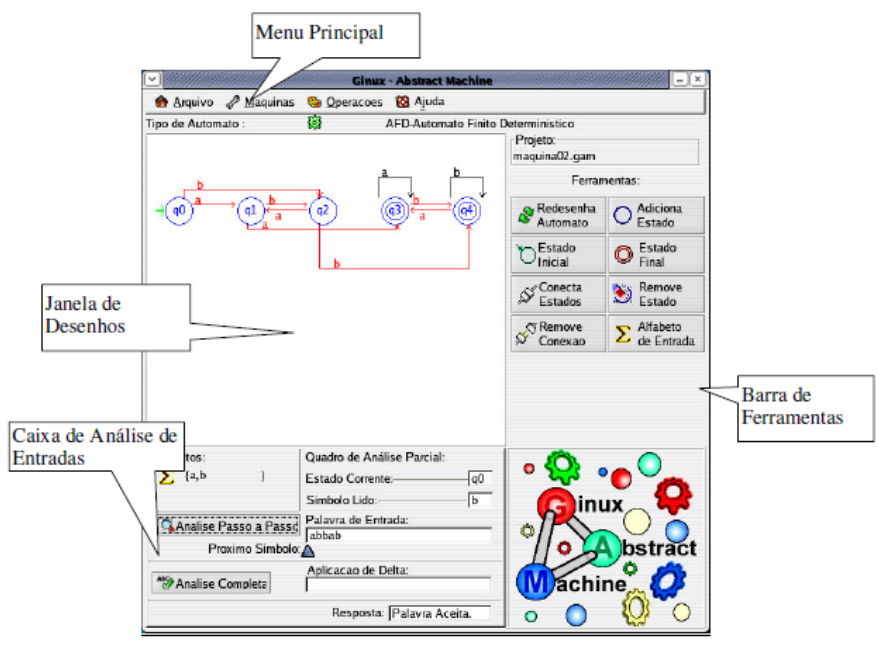

Figura 1: Interface principal de GAM [1]

A segunda ferramenta computacional apresentada na Tabela 1 é a Visual Automata Simulator (VAS) [2]. Ela permite o estudo de um modelo computacional a mais do que a GAM [1]: a Máquina de Turing (MT). Suas interfaces gráficas permitem ao usuário visualizar e manipular estados e transições diretamente, enquanto se criam AFD, AFND ou MT como solução a um exercício. A VAS [2], embora permita a conversão de AFND em AFD, não executa o processo de minimização de AFDs. O ponto forte da VAS é sua manipulação de MT: a interface permite uma manipulação diferente das demais ferramentas, pois possui botões que facilitam o processo de ida e volta realizada pela cabeça de leitura sobre a fita no processamento da máquina e uma visualização da fita após o processo de reconhecimento ter sido completado. Na Figura 2 a marcação em vermelho rotulada como (1) representa o uso do botão do menu que retorna a cabeça de leitura da MT até o símbolo “\#”. 


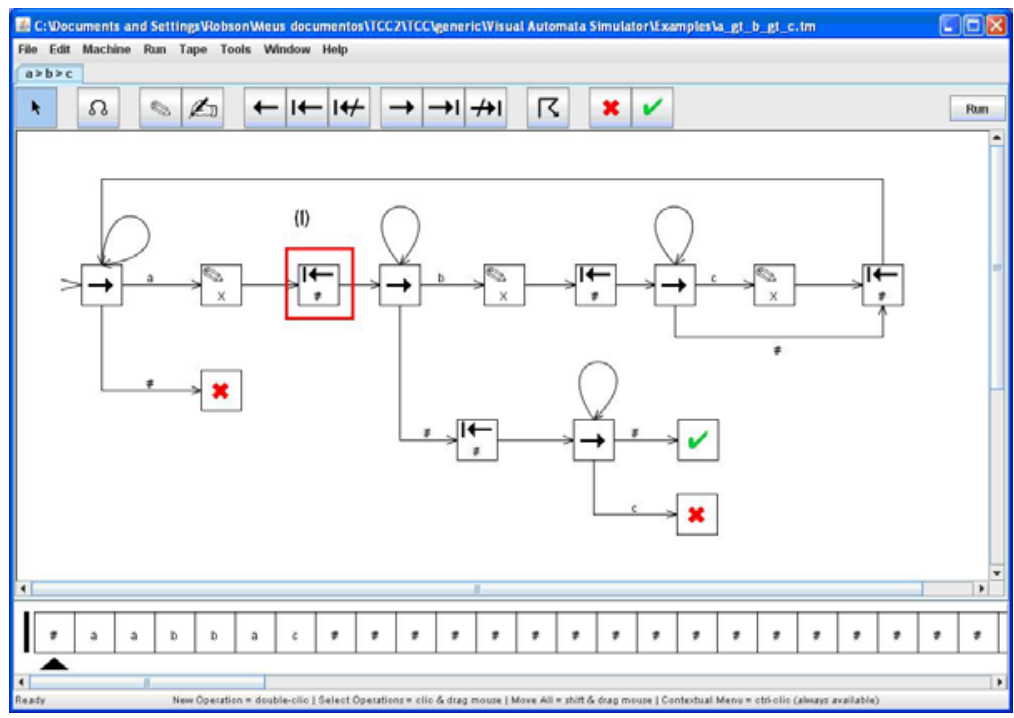

Figura 2: Interface para MT no VAS [2].

A terceira linha da Tabela 1 apresenta o Language Emulator [4]. Essa ferramenta proporciona ao usuário o estudo de um maior número de modelos computacionais do que GAM [1] e VAS [2] a saber: AFD, AFND (dividido em AFNDs sem transição vazia "AFN" e com transição vazia "AFN-Lambda”), GRs, ERs, Máquinas de Moore e Máquinas de Mealy, porém não implementa MT.

Embora não implemente MT, o Language Emulator permite realizar várias conversões entre modelos computacionais equivalentes, por exemplo, entre Máquinas de Moore e Máquinas de Mealy. Seu grande diferencial é a forma analítica de validar uma palavra: exibe todos os passos de processamento do modelo computacional criado durante o processo de reconhecimento. Pode ser indicado por professores a alunos com dificuldades na definição formal de modelos computacionais.

A Figura 3 apresenta em vermelho os campos para definição dos estados, alfabeto, estado inicial e o conjunto de estados finais para um AFD em (1), a definição da função de transição em (2), um campo para inserção de palavras de teste em (3), botões para minimização e conversão para modelos equivalentes em (4) e os passos de processamento da ferramenta ao se criar o modelo em (5).

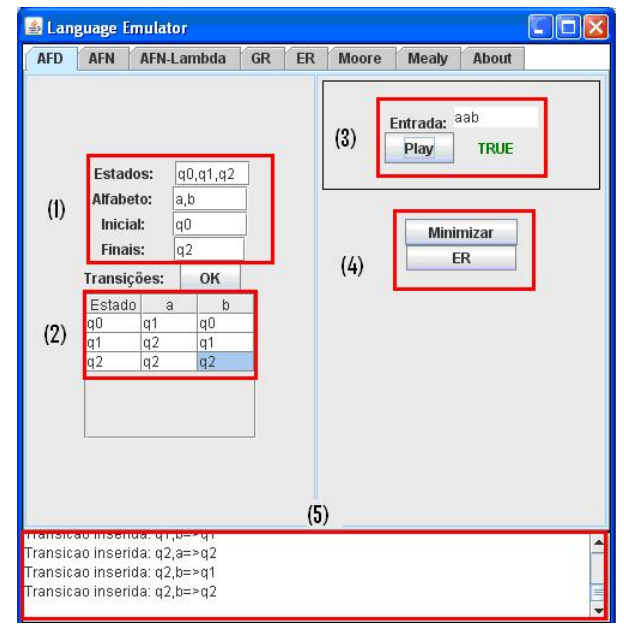

Figura 3: Interface Language Emulator [4] e transições construídas

O Sistema de Criação e Teste de Modelos Formais (SCTMF) [3] é similar à ferramenta computacional Language Emulator [4] no momento em que ajudam o aluno a superar dificuldades em definir formalmente modelos computacionais. Seu diferencial está no processo de interação do usuário com o sistema em que são usadas interfaces separadas para definir o alfabeto, outra para o conjunto de estados, uma terceira para as transições e uma última para testes com palavras de teste. A Figura 4 exibe a tela para reconhecimento de palavras. Note que a partir das definições anteriores o modelo formal vai sendo construído e pode ser visto na parte marcada em vermelho e assinalada com o rótulo (1) à esquerda 


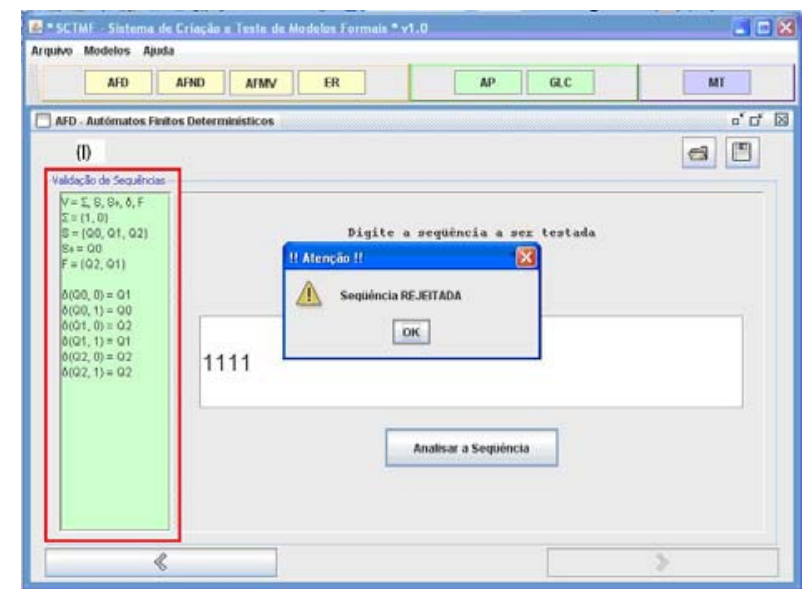

Figura 4: Interface para reconhecimento de palavras no SCTMF [3].

A quinta linha da Tabela 1 apresenta o Java Formal Languages and Automata Package (JFLAP) [5], outra ferramenta computacional para ajudar os alunos em disciplinas de TC. Dentre todas as ferramentas computacionais apresentadas, ela é a mais completa. Além de implementar AFDs e AFNDs como todas as outras quatro ferramentas, também permite o estudo de MT com múltiplas fitas, Gramáticas e ERs e mais recentemente Máquinas de Moore e Mealy.

Os pontos fortes da JFLAP [5] são: o processo de reconhecimento de palavras passo a passo (o aluno acompanha todos os passos envolvidos no reconhecimento de uma palavra pelo modelo computacional desenhado, em vez de simplesmente ser informado que a palavra foi aceita ou rejeitada), a variedade implementada de conversões de modelos computacionais em seus equivalentes e a possibilidade de visualizar e manipular diretamente os estados e as transições dos modelos computacionais criados. A Figura 5 exibe a tela para a construção do modelo formal AFND no JFLAP [5].

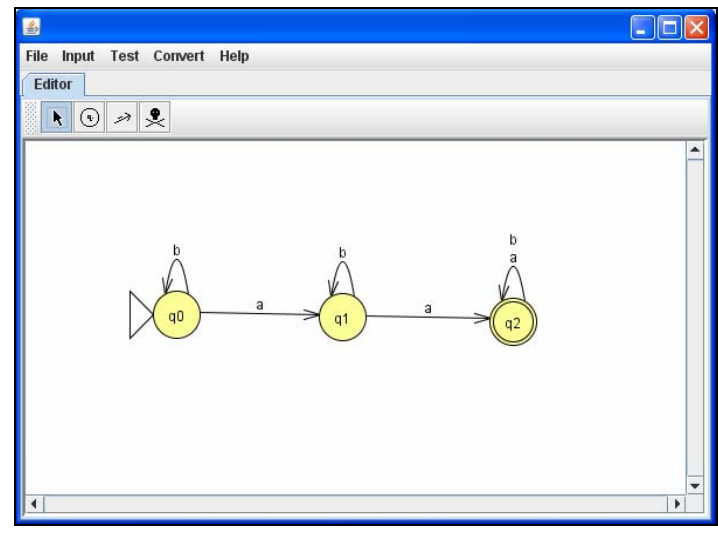

Figura 5: Interface para AFND no JFLAP [5].

Acredita-se que mesmo com todas essas ferramentas computacionais disponíveis (GAM [1], VAS [2], Language Emulator [4], SCTMF [3] e JFLAP [5]), muitas disciplinas podem apresentar resultados pouco satisfatórios, a exemplo do que ocorre na UFPA. Isto porque os alunos realmente têm dificuldades em assimilar fundamentos de TC como abordados em [3,7,8,9].

A Figura 6 apresenta dados relativos à reprovação em ofertas da disciplina de Linguagens Formais entre o segundo semestre de 2005 e o segundo semestre de 2009 na UFPA por diferentes professores. Eles mostram que pelo menos 30\% dos alunos não conseguem atingir o conceito mínimo para aprovação na disciplina. Nesse contexto, vários podem ser os fatores que ocasionam esse resultado. Um deles acredita-se ser a não resolução das listas de exercícios propostas pelo professor ao longo da disciplina.

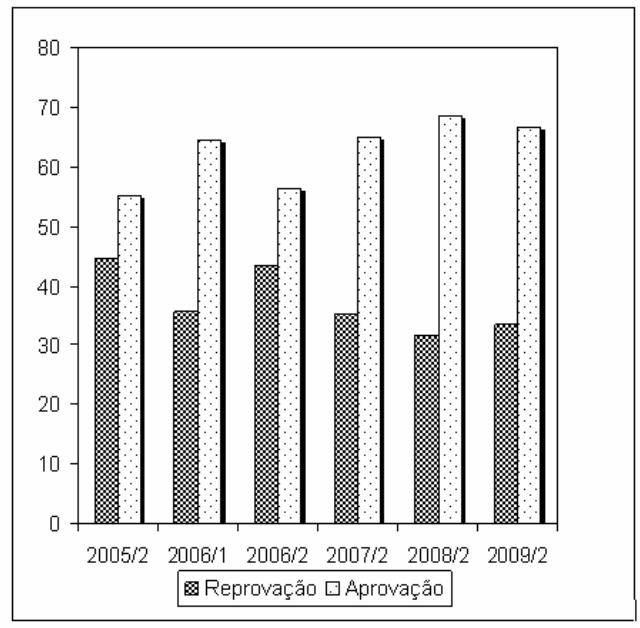

Figura 6: Percentual de reprovação e aprovação em Linguagens Formais.

Considerando as dificuldades do aluno em compreen- 
der os conceitos de TC por seus caráteres abstratos e matemáticos $[7,8]$ e principalmente de saber rapidamente se sua solução a um exercício proposto pelo professor em listas está correta ou não, o LabLF foi construído para auxiliar o aluno nessas dificuldades específicas.

O LabLF é um ambiente computacional que permite o estudo dos modelos computacionais AFD, AFND, GR, ER, AP e MT. Seu diferencial reside na possibilidade de armazenar gabaritos para correção automática ou semiautomática de alguns exercícios de TC, elaborados pelo professor e disponibilizados online via Web para os alunos. A seção seguinte (seção 3) apresenta o LabLF, abordando a sua implementação, a criação e correção automática e semi-automática de exercícios.

\section{Laboratório de Linguagens For- mais (LabLF)}

A ferramenta adotada por professores da UFPA para dar suporte aos seus cursos de TC foi o JFLAP. Como dito anteriormente, essa ferramenta computacional, assim como outras, permite ao aluno resolver exercícios e fornecer palavras de teste para averiguar quais, seu modelo computacional, aceita e quais rejeita. Porém, os alunos desejavam saber rapidamente se suas soluções estavam corretas ou não, logo poderia ocorrer o fornecimento de palavras de teste inadequadas para que se possa afirmar realmente que a solução está correta.

Aliado a isto, os diagramas de modelos computacionais solicitados como resposta às perguntas de avaliações podem variar em forma e tamanho devido ao processo criativo de construção do mesmo por cada aluno. Assim, a tarefa de correção de avaliações é um processo demorado.

Neste contexto, com o desenvolvimento do ambiente LabLF busca-se auxiliar: o aluno, propondo um processo automatizado e semi-automatizado para correção de exercícios de listas, que permita a ele saber rapidamente se sua solução está correta ou não; e o professor, a postar materiais, criar listas de exercícios e corrigir perguntas de avaliações com os processos de correção automático ou semi-automático.

As subseções a seguir (3.1, 3.2 e 3.3) apresentam, respectivamente, uma visão geral do desenvolvimento do LabLF, o processo de criação de exercícios e a correção automática e semi-automática de exercícios.

\subsection{Uma visão do desenvolvimento do LabLF}

As ferramentas computacionais GAM [1], VAS [2], Language Emulator [4], SCTMF [3] e JFLAP [5] apresentadas previamente na seção 2 têm em comum a linguagem utilizada em seus desenvolvimentos: Java. Além disso, todas são ferramentas desktop em que o aluno pode baixá-las e executá-las em seu computador.

Por outro lado, o LabLF é um dos ambientes computacionais do tipo cliente/servidor desenvolvido em PHP e comunicação com Banco de Dados (BD) MySQL de um conjunto de ambientes voltados aos estudos de várias disciplinas ofertadas na UFPA.

O LabLF reutiliza editores da ferramenta computacional JFLAP [5]. Sua escolha se deu pelo fato, que dentre as ferramentas computacionais pesquisadas e apresentadas anteriormente é a que possui o maior número de modelos computacionais e conversões entre modelos equivalentes implementados e código livre.

Os editores do JFLAP [5] foram reutilizados dentro do LabLF para inserção de modelos computacionais como respostas a exercícios de TC. Também foram aproveitadas suas funcionalidades de conversão e teste de palavras para corrigir automaticamente ou semiautomaticamente tais exercícios.

A implementação do LabLF utilizou a linguagem de programação PHP que já estava em uso na implementação de outros laboratórios virtuais como o LabSQL [12]. A primeira tarefa foi, portanto, embutir os editores do JFLAP [5] escritos em Java nas páginas em PHP do LabLF. Este problema foi solucionado exibindo a aplicação Java como applets dentro do sistema web proposto, através do uso das tags <applet $><$ /applet $>$ da linguagem PHP.

Posteriormente, outro problema encontrado foi em como armazenar no BD do LabLF os modelos computacionais construídos graficamente pelo usuário nos editores do JFLAP embutidos nas páginas PHP. A solução consistiu em modificar o código da ferramenta inserindo métodos para conversão do modelo computacional apresentado graficamente em String e utilizar a passagem de parâmetros das tags <applet></applet> do PHP para capturar a resposta destes métodos e inserir o modelo computacional em forma textual no BD do LabLF. Assim, foi necessário construir esses métodos de conversão para todos os modelos computacionais utilizados no LabLF além dos métodos para converter modelos computacionais armazenados em BD como Strings em suas representações diagramáticas.

Após realizadas essas tarefa buscou-se utilizar outros recursos do JFLAP, como a conversão de modelos computacionais em seus equivalentes e de testes de palavras, para implementar a correção automática de alguns tipos de exercícios e a semi-automática para outros.

Assim, nos exercícios relacionados à criação de modelos computacionais que possuem equivalência entre máquinas (AFND, AFD, GR e ER), a solução dada pelo 
usuário passa internamente pelos processos de conversão em AFD e minimização deste, chegando a um AFD que é único por definição [10]. Após essas conversões, o LabLF pode corrigi-los automaticamente.

Já para os exercícios onde suas soluções são os modelos AP ou MT, que não possuem equivalência entre máquinas, são corrigidos de maneira semi-automática, isto é, são realizadas verificações com palavras de testes, necessitando posteriormente revisão desta correção.

Além destes exercícios também são corrigidos automaticamente os exercícios de Verdadeiro ou Falso e os de Múltipla Escolha que possuem respostas objetivas. Já para o tipo de exercício Discursivo do LabLF não há quaisquer recursos para automatização de sua correção, por fugir do escopo da abordagem deste trabalho. Porém nada impede que futuramente técnicas de processamento de palavras e detecção de semântica sejam implementados para facilitar seu processo de correção.

\subsection{A criação de exercícios}

O LabLF permite a criação de diferentes tipos de exercícios que são amplamente conhecidos e por essa razão não serão discutidos neste artigo (Discursivo, Verdadeiro ou Falso e de Múltipla Escolha). Além desses tipos, como dito anteriormente, é possível criar exercícios que explorem a criação dos seguintes modelos computacionais: AFND, AFD, GR, ER, AP e MT.

Para exemplificar o processo de criação de um exercício no LabLF é necessário que o leitor saiba que são quatro os tipos de usuários do ambiente: Gerente (administrador), Professor, Monitor (também pode corrigir avaliações) e Aluno. Dentre esses perfis, o usuário "Professor” será usado para mostrar os passos de criação de um exercício livre ${ }^{1}$ de AFND no LabLF.

A Figura 7 exibe a tela para criação de um exercício livre de AFND no LabLF por um usuário do tipo professor. Os campos a serem preenchidos são:

- "Enunciado" (onde se lê "Considerando o alfabeto $\{0,1\}$ construa o diagrama de um Autômato Finito Não-Determinístico que aceite todas as palavras tal que todo 0 apareça entre dois 1s.”) com a descrição de um problema que deve ser solucionado com um AFND,

- "Resposta” com o diagrama de um AFND que é solução do exercício e

- "Palavras de teste” para inserção das palavras a serem testadas sob o diagrama do AFND

\footnotetext{
${ }^{1}$ Exercício livre é todo exercício elaborado pelo professor e disponibilizado aos alunos em que sua solução é um dos seguintes modelos computacionais: AFND, AFD, GR, ER, AFP e MT.
}

construído e inserido no campo "Resposta”.

Após o preenchimento de todos os campos, o professor pode disponibilizar o exercício para seus alunos clicando no botão "Submeter" ou desistir de cadastrar tal exercício simplesmente clicando em "Voltar”.

É desta forma que todos os tipos de exercícios livres implementados no LabLF (AFND, AFD, GR, ER, AP e MT) são criados pelo professor e disponibilizados a seus alunos.

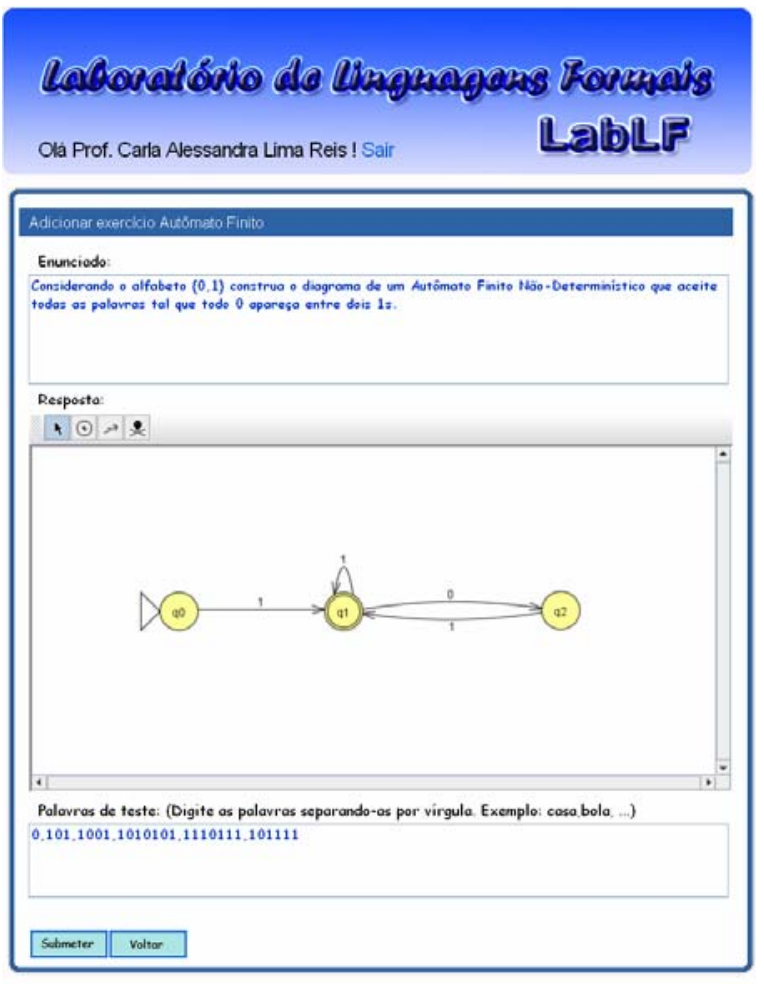

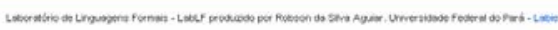

Figura 7: Exercício livre de AFND no LabLF

\subsection{Correção automática e semi-automática de exercícios livres}

Até este momento vem se comentando sobre o processo de correção automática e semi-automática de exercícios livres, que podem ajudar o aluno a saber rapidamente se sua solução a um exercício livre proposto pelo professor está correta ou não.

Assim, para exemplificar o processo de correção automático de exercícios livres, a Figura 8 apresenta a tela exibida pelo LabLF quando um aluno ("z") acessa o ambiente e tenta responder o exercício livre cadastrado pelo professor visto na Figura 7. Nessa tela são exibidos:

- O enunciado do exercício;

- O editor correspondente ao modelo a ser cons- 
truído (editor para construir AFNDs);

- Um campo para o aluno inserir uma palavra de teste e verificar, por meio do botão "Testar", se a sua solução a aceita ou a rejeita;

- O botão "Voltar” para desistir de submeter sua solução à correção; e

- O botão “Submeter”, para enviar sua resposta para correção, no caso do exemplo, automática.

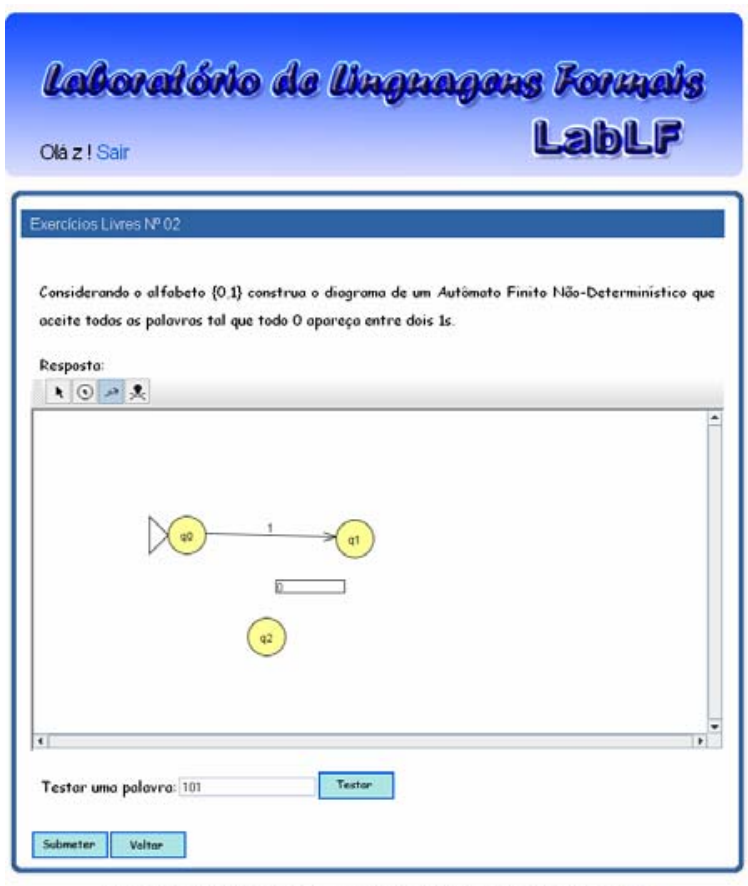

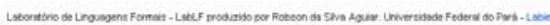

Figura 8: Exercício Livre de AFND no LabLF.

Após clicar no botão "Submeter" o aluno é direcionado a uma página que o informa rapidamente se sua solução é a esperada pelo professor ou não.

Assim, a tela exibida na Figura 9 é apresentada caso a solução pré-cadastrada pelo professor no ato de criação do exercício e a solução submetida pelo aluno convirjam para o mesmo AFD mínimo.

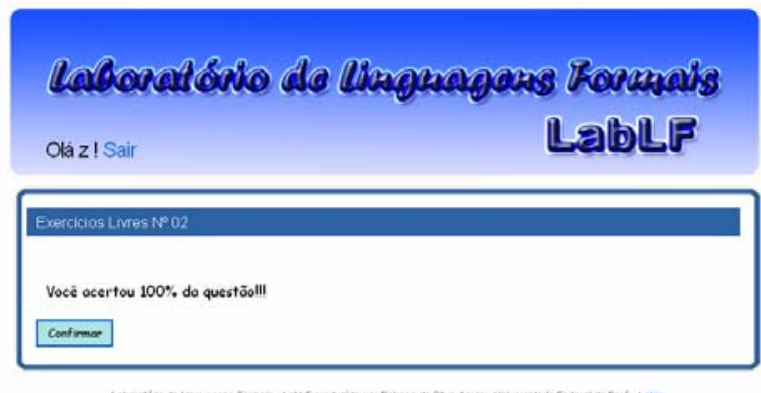

Figura 9: Feedback gerado ao aluno informando acerto de exercí- cio.

A Figura 10 apresenta o feedback gerado automaticamente pelo LabLF quando a solução do aluno não é a solução pré-cadastrada e esperada pelo professor. Assim, diferentemente das ferramentas computacionais discutidas neste trabalho (GAM [1], VAS [2], SCTMF [3], Language Emulator [4] e JFLAP [5]) o LabLF calcula um percentual de acerto da solução do aluno em relação à solução do professor com base nos AFDs mínimos de suas soluções e, adicionalmente informa ao aluno palavras que deveriam ser aceitas e não foram pelo modelo computacional dado como resposta ao exercício e palavras que não deveriam ser aceitas e foram, como pode ser visto na última linha deste feedback "O autômato finito não deveria reconhecer a(s) palavra(s): 0”.

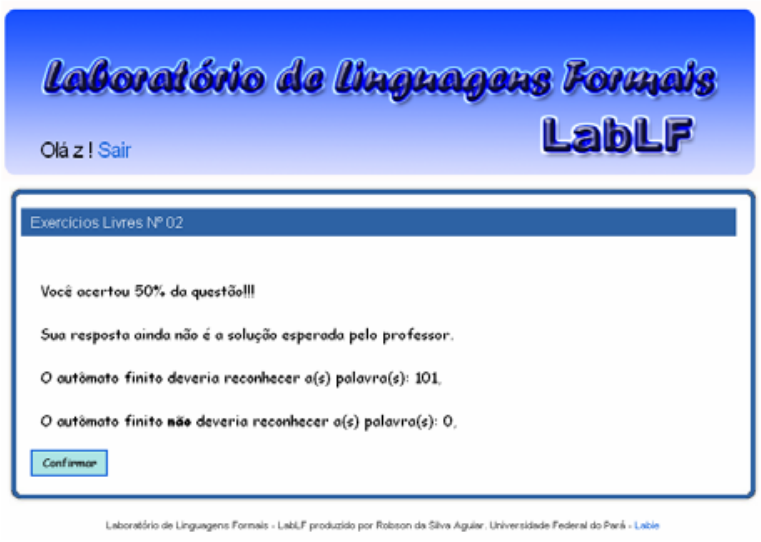

Figura 10: Feedback gerado ao aluno informando erro no exercício.

A seguir, a seção 4 discorre sobre o uso do LabLF em uma turma real na UFPA.

\section{0 uso do LabLF}

Antes do desenvolvimento do LabLF e sua utilização pela primeira vez na disciplina de Linguagens Formais do $2^{\circ}$ Semestre de 2009 (LF-2009/2) da UFPA, o planejamento destas disciplinas estabelecia as seguintes atividades:

- Um conjunto de assuntos seriam abordados em sala pelo professor por meio de aulas teóricas;

- Aulas de “tirar dúvidas” em sala de aula;

- Duas avaliações (provas) seriam realizadas durante o semestre; e

- Um seminário seria realizado pelos alunos apresentando aplicações dos assuntos estudados em TC a problemas reais.

Porém, com o desenvolvimento do LabLF este plane- 
jamento da disciplina teve que ser alterado. Para a disciplina que ocorreu em 2009/2, além das atividades descritas anteriormente, foi estabelecido um conjunto de três trabalhos a serem realizados no LabLF ao longo da disciplina.

Basicamente, estes trabalhos foram a maneira encontrada pelo professor para que os alunos tivessem contato com os processos de correção automática e semiautomática implementados no LabLF. Assim, no trabalho:

1. Os alunos tiveram contato com o processo de correção automática de exercícios livres de AFD e AFND;

2. Os alunos foram submetidos a exercícios livres de AP com correção semi-automática; e

3. Os exercícios livres abordaram problemas em que MTs deveriam ser construídas com soluções.

A partir destes trabalhos se observou dois pontos importantes:

$1^{\circ}$. Os alunos a partir dos feedbacks gerados pelo LabLF buscavam atingir sempre $100 \%$ de acerto do exercício; e

$2^{\circ}$. Alguns alunos conseguiram perceber que o professor cadastrou uma solução incompleta a um exercício livre.

Em relação a este último ponto, embora não tenha sido proposital o cadastro de uma solução parcial a um exercício livre pelo professor, serviu para observar que alguns alunos conseguiram perceber uma limitação da resposta dada pelo professor.

Atualmente, dado o grande número de instituições de ensino nacionais e internacionais que utilizam o Moodle [11], uma delas a UFPA, decidiu-se que o LabLF migraria para o Moodle [11] em 2010. Assim, está em teste na UFPA, na disciplina de Linguagens Formais do $2^{\circ}$ Período Letivo de 2010 (LF-2010/2), um ambiente Moodle [11] que simula o LabLF.

Para isso foram efetuadas modificações no Moodle "padrão" a fim de incluir os tipos de exercícios de TC descritos anteriormente. A Figura 11 apresenta a tela exibida ao professor para criar um exercício de AFD no Moodle [11] que simula o LabLF, onde:

- O enunciado do exercício (1);

- A solução do exercício (2); e

- As palavras de teste (3).

A Figura 12 apresenta a tela que é exibida ao aluno para submissão de uma solução a um exercício proposto pelo professor. Nela o aluno pode:

- Ler o enunciado do exercício (1);

- Inserir a sua resposta (2); e

- Submeter sua solução à correção automática clicando no botão "Submeter" ou cancelar a correção, clicando no botão "Cancelar” (3). 


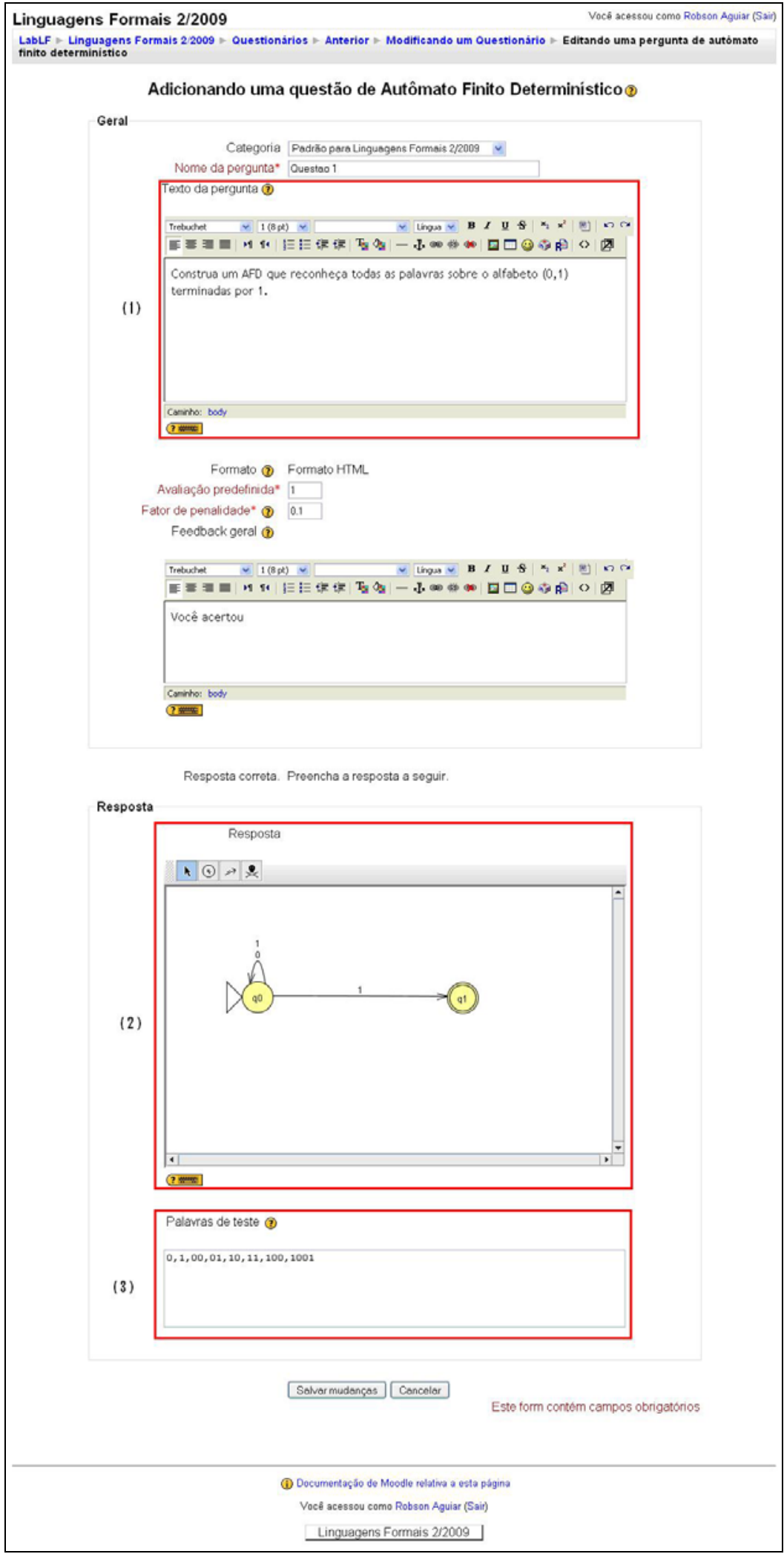

Figura 11: Tela Moodle [11] para criação de um exercício de AFD. 


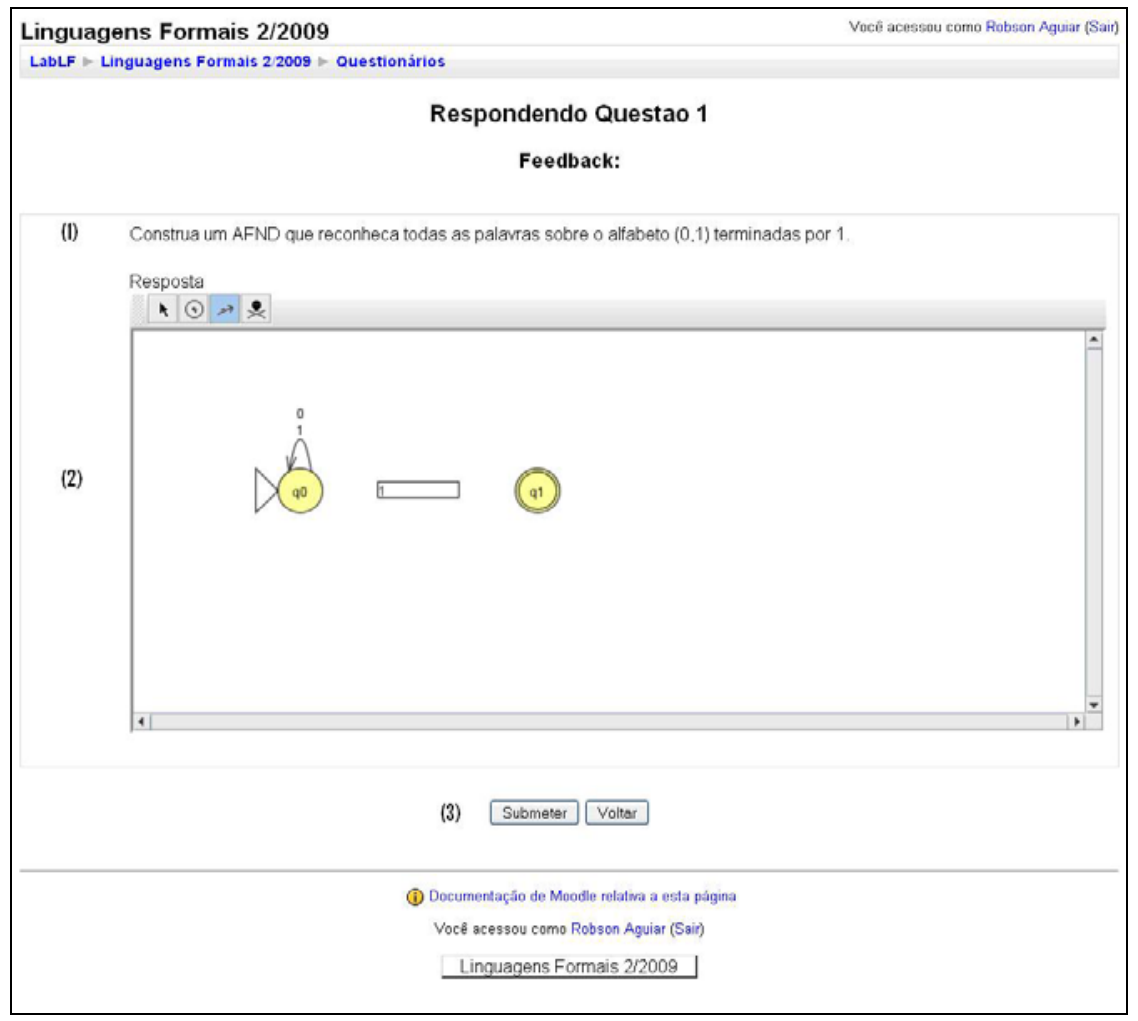

Figura 12: Tela para submissão de resposta de exercício pelo aluno no Moodle [11].

As Figuras 13 e 14 apresentam, respectivamente, os feedbacks exibidos ao aluno após submeter uma solução que ainda não é a solução esperada pelo professor ou a que corresponde a esperada por este.

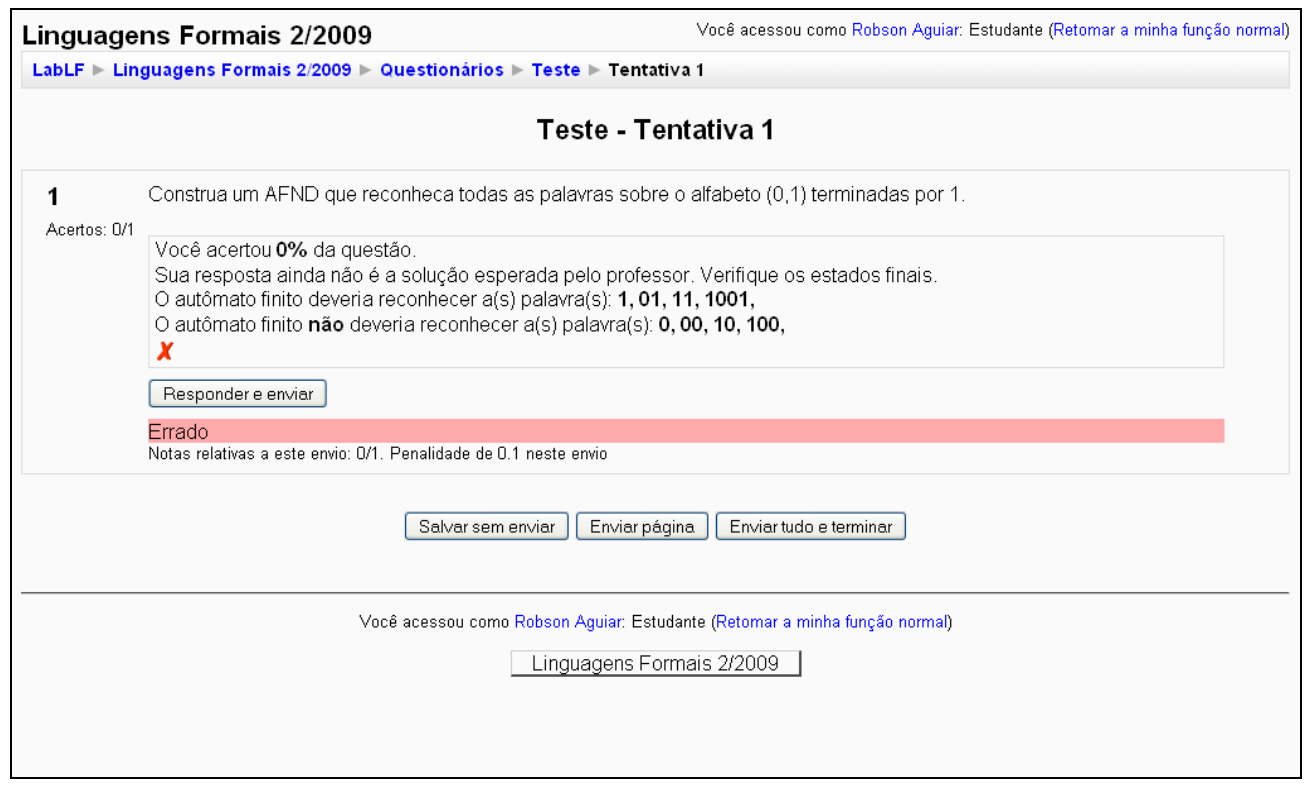

Figura 13: Feedback de solução não esperada pelo professor 


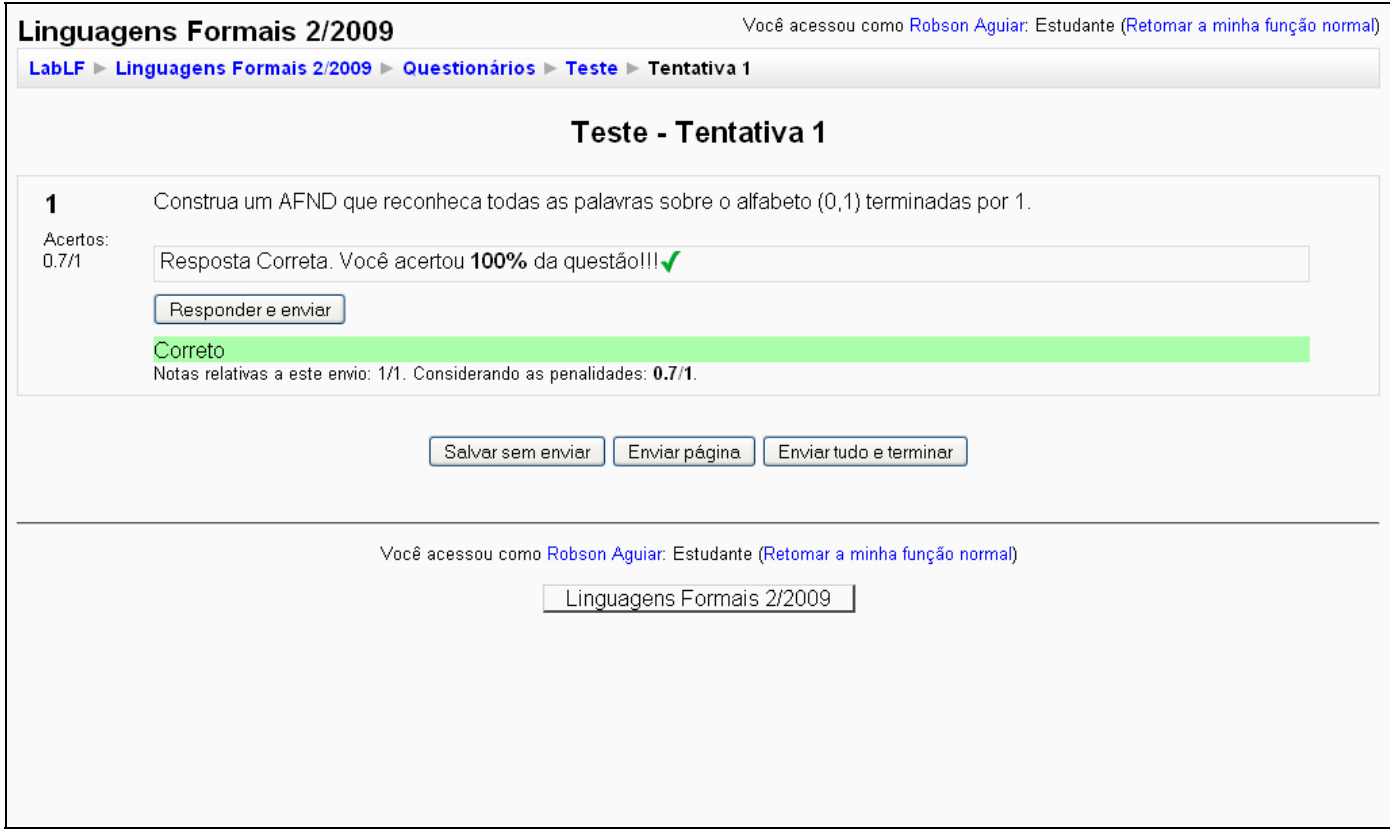

Figura 14: Feedback de solução esperada pelo professor.

É importante ressaltar que houve uma mudança nas atividades planejadas para a disciplina LF-2010/2 para permitir o uso Moodle [11] que simula o LabLF. Assim, ficou estabelecido que:

- Um conjunto de assuntos seriam abordados em sala pelo professor;

- As duas avaliações seriam desmembradas em seis avaliações;

- Um seminário deve ser realizado com aplicações dos assuntos estudados em TC a problemas reais; e

- Algumas aulas seriam destinadas a realização de exercícios em laboratório neste ambiente Moodle [11] com adaptações que simulam o LabLF.

Assim, o que se tem observado a partir de entrevistas com os alunos de LF-2010/2 (18 alunos inscritos, mas somente 12 comparecem às aulas e estão realizando as avaliações), em que 6 alunos estão fazendo a disciplina por pelo menos mais de uma vez e 6 pela primeira vez, é que:

- $100 \%(6 / 6)$ dos alunos que fazem pela primeira vez e $83,33 \%(5 / 6)$ dos alunos que estão fazendo a disciplina por pelo menos mais de uma vez afirmam que a verificação pelo ambiente de uma solução a um problema pro- posto pelo professor os ajuda muito em sua aprendizagem;

- 66,67\% (4/6) dos alunos que estão fazendo a disciplina por pelo menos mais de uma vez afirmam que o feedback gerado na correção automática e semi-automática os ajudam em parte a saberem se suas soluções estão corretas;

- 33,33\% (2/6) dos alunos que estão fazendo a disciplina por pelo menos mais de uma vez e que experimentaram duas avaliações no semestre anterior e seis miniavaliações neste semestre relataram como ponto forte o menor número de assuntos a serem estudados para cada uma das miniavaliações e

- 33,33\% (2/6) dos alunos que estão fazendo a disciplina por pelo menos mais de uma vez afirmam que as miniavaliações permitem a assimilação maior de informações mais facilmente.

A seguir na seção 5 são apresentadas as considerações finais do trabalho.

\section{Considerações Finais}

O LabLF foi criado para ser utilizado tanto por professores e alunos, por meio do cadastro e da resolução de exercícios e de avaliações. Com a sua utilização, esperou- 
se dinamizar e facilitar a aprendizagem de Teoria da Computação, estimulando os alunos a encontrarem, por meio do feedback fornecido, a solução para os exercícios propostos pelo professor ao longo da disciplina.

No que diz respeito ao professor, a ferramenta pode ajudá-lo na tarefa de correção de avaliações de uma forma mais imparcial (correção automática e semiautomática) e também a acompanhar o desempenho dos alunos (quais fizeram as listas, quantas questões fizeram) e assim poder tomar atitudes ao longo da disciplina visando o sucesso de todos os alunos.

Devido o LabLF permitir que os assuntos de Teoria da Computação possam ser explorados via simulações e visualizações pelos alunos e tornem a vida daquele que aprende e daquele que ensina facilitada, buscou-se integrá-lo ao ambiente Moodle [11].

Atualmente, este ambiente Moodle com adaptações que simulam o LabLF está em teste em LF-2010/2 e já se percebe que algumas modificações devem ser realizadas futuramente como: o aperfeiçoamento do feedback para informar com mais precisão o erro ao aluno e o melhoramento de interfaces e sinalizações do Moodle [11]. Além disso, outros testes precisam ser feitos como em: navegadores diferentes do Mozilla Firefox e BD PostgreSQL.

\section{Referências}

[1] A. S. Jukemura, A. D. Hugo, J. Q. Uchoa. GAM - Um Simulador para Auxiliar o Ensino de Teoria da Computação. In Anais do XXV Congresso da Sociedade Brasileira de Computação, São Leopoldo, páginas 2432-2443, 2005.

[2] J. Bovet. Visual Automata Simulator: A tool for simulating, visualizing and transforming finite state automata and Turing Machines. http://www.cs.usfca.edu/ jbovet/vas.html, Out. 2008.

[3] Y. M. G. Costa, R. C. Meneses, F. R. Uber. Uma Ferramenta para Auxílio Didático no Ensino de Teoria da Computação. In Anais do XXVIII Congresso da Sociedade Brasileira de Computação, Belém, páginas 208-217, 2008.

[4] N. J. Vieira. Fundamentos da Teoria da Computação.

http://homepages.dcc.ufmg.br/ lfvieira/ftc.html, Dez. 2008.

[5] S. H. Rodger. JFLAP Version 7.0: RELESEAD August 28, 2009. http://www.jflap.org, Out. 2009.
[6] A. O. Bilska, K. H. Leider, M. Procopiuc, O. Procopiuc, S. H. Rodger, E. Tsang. A Collection of Tools for Making Automata Theory and Formal Languages Come Alive. In Anais do SIGCSE ‘97, San Jose, páginas 15-19, 1997.

[7] M. Goyal, S. Sachdeva. Enhancing Theory of Computation Teaching Through Integration with other Courses. In Anais do International Journal Of Recent Trends In Engineering, Oulu, páginas 137-140, 2009.

[8] C. I. Chesñevar, A. G. Maguitman, M. P. González, M. L. Cobo. Teaching Fundamentals of Computing Theory: A Constructivist Approach. In Anais do Journal Of Computer Science \& Technology: JCS\&T, Espanha, páginas 91-97, 2004.

[9] E. Gramond, S. H. Rodger. Using JFLAP to interact with theorems in automata theory. In Anais do SIGCSE '99, New Orleans, páginas 336-340, 1999.

[10] P. B. Menezes. Linguagens Formais e Autômatos. Sagra Luzzatto, Porto Alegre, página 66, 2000.

[11] Moodle. http://moodle.org/, Mar. 2010.

[12] A. D. P. Lino, A. S. Silva, T. L. T. Santos, M. P. A. H. Harb, E. L. Fávero, S. R. Brito. Avaliação automática de consultas SQL em ambiente virtual de ensino-aprendizagem. In Anais do CISTI 2007, Porto, 2007. 